\title{
HOLOMORPHIC FIBER BUNDLES WITH HYPERBOLIC FIBER ${ }^{1}$
}

\author{
H. L. ROYDEN
}

\begin{abstract}
The purpose of the present note is to show that holomorphic fiber bundles with hyperbolic fiber are almost trivial: They are trivial if the base is simply connected, and have a simple structure otherwise.
\end{abstract}

Kobayashi [2], [3] has introduced the notion of a hyperbolic complex manifold. We need not be concerned with the definition here, but only with the following consequence [3], [4]: If $M$ is a hyperbolic complex manifold, $p$ a point of $M$, and $U$ a neighborhood of $p$, then there is a $\delta>0$ such that any holomorphic map $\phi$ of the unit disk into $M$ with $\phi(0)=p$ maps the disk $\{z:|z|<\delta\}$ into $U$. From this, it follows that, if $w^{1}, \cdots, w^{n}$ are coordinates at $p$, and $l$ an integer, then there are constants $A_{l}$ depending only on $l$ and the choice of coordinates such that, if $\phi$ is any map of the unit disk into $M$ with $\phi(0)=p$, then $\left|\partial^{l} w^{i} / \partial z^{l}\right|<A_{l}$ at $z=0$.

The crux of our argument is the following proposition, which generalizes a result of Kobayashi's that a complex Lie group (of dimension greater than 0 ) cannot act holomorphically on a hyperbolic manifold.

Proposition. Let $M$ and $N$ be complex manifolds with $M$ hyperbolic. Let $F=F(w, z)$ be a holomorphic map of $M \times N$ into $M$. Suppose that, for some $z_{0} \in N$, the restriction of $F$ to $M \times\left\{z_{0}\right\}$ is a biholomorphic map onto $M$. Then $F$ is independent of $z$; i.e., $F(w, z)=F\left(w, z_{0}\right)$.

Proof. It suffices to prove the proposition for the case that $N$ is a ball, and hence for the case that $N$ is a disk $\Delta$, which we take to be the unit disk with $z_{0}=0$. Without loss of generality, we may assume $F\left(w, z_{0}\right)=w$. Near a point $\left\langle w_{0}, z_{0}\right\rangle$, the map $F$ has a power series expansion of the form

$$
F^{i}(w, z)=w^{i}+a^{i}(w) z^{l}+O\left(z^{l+1}\right) .
$$

Received by the editors February 26, 1973 and, in revised form, June 6, 1973.

AMS (MOS) subject classifications (1970). Primary 32H20, 32L05.

Key words and phrases. Holomorphic fiber bundles, hyperbolic manifolds, Kobayashi, hyperbolicity, tight manifolds.

${ }^{1}$ This work partially supported by NSF Grant GP-33492 X.

(c) American Mathematical Society 1974 
We inductively define maps $\phi_{k}$ of the unit disk into $M$ by setting $\phi_{0}=w_{0}$ and $\phi_{k}(z)=F\left(\phi_{k-1}(z), z\right)$. Then near $z=0$, the map $\phi_{k}$ has the expansion

$$
\phi_{k}^{i}(z)=w_{0}^{i}+k a^{i}\left(w_{0}\right) z^{l}+O\left(z^{l+1}\right) .
$$

Since $M$ is hyperbolic, there is a constant $A_{l}$ independent of $k$ such that

$$
l !\left|k a^{i}\left(w_{0}\right)\right|=\left|\frac{\partial^{l} \phi_{k}^{i}}{\partial z^{l}}\right|<A_{l} .
$$

Hence $a^{i}\left(w_{0}\right)=0$. But this means that the derivatives of $F$ of all orders with respect to $z$ vanish at $\left\langle w_{0}, z_{0}\right\rangle$. Since $w_{0}$ was an arbitrary point of $M$, we must have $F$ independent of $z$, proving the proposition.

As an immediate consequence, we have the following two corollaries:

COROLlaRY 1. A holomorphic fiber bundle with simply connected base and hyperbolic fiber is trivial.

COROLlaRY 2. The holomorphic fiber bundles with hyperbolic fiber $M$ and base $B$ are in natural one-to-one correspondence with the homomorphisms of the fundamental group of $B$ into the group of biholomorphic automorphisms of $M$ onto itself.

If we make use of the facts that the product of two (complete) hyperbolic manifolds is (complete) hyperbolic and that a manifold is (complete) hyperbolic iff its universal covering manifold is [3], [4], we obtain from Corollary 1 the following result of Kiernan [1]:

COROllary 3. A holomorphic fiber bundle with (complete) hyperbolic fiber and base is (complete) hyperbolic.

\section{BIBLIOGRAPHY}

1. P. Kiernan, Results concerning hyperbolic manifolds, Proc. Amer. Math. Soc. 25 (1970), 588-592. MR 41 \#2044.

2. S. Kobayashi, Invariant distances on complex manifolds and holomorphic mappings, J. Math. Soc. Japan 19 (1967), 460-480. MR 38 \#736.

3. - Hyperbolic manifolds and holomorphic mappings, Pure and Appl. Math., vol. 2, Dekker, New York, 1970. MR 43 \#3503.

4. H. L. Royden, Remarks on the Kobayashi metric, Proc. Maryland Conf. on Several Complex Variables, Lecture Notes in Math., vol. 185, Springer-Verlag, Berlin and New York.

Department of Mathematics, Stanford University, Stanford, California 94305 